\title{
Image Reconstruction for High-Performance Electrical Capacitance Tomography System Using Deep Learning
}

\author{
Yanpeng Zhang and Deyun Chen (iD \\ School of Computer Science and Technology, Harbin University of Science and Technology, Harbin, Heilongjiang 150080, China \\ Correspondence should be addressed to Deyun Chen; deyunchen_1962@126.com
}

Received 2 March 2021; Revised 11 April 2021; Accepted 20 April 2021; Published 3 July 2021

Academic Editor: M. Irfan Uddin

Copyright (c) 2021 Yanpeng Zhang and Deyun Chen. This is an open access article distributed under the Creative Commons Attribution License, which permits unrestricted use, distribution, and reproduction in any medium, provided the original work is properly cited.

\begin{abstract}
For great achievements in recent decades, image reconstruction for electrical capacitance tomography (ECT) has been considered in this study. ECT has demonstrated impressive potentials in multiprocess measurement, and the obtained images are of high resolution, which are suitable for advanced procedures in industrial and medical applications and across different tasks and domains. But the ECT system still requires improvements in the quality of image reconstruction given its importance of great significance to obtain the reliability and usefulness of measurement results. The deep neural network is used in this study to extract new features and to update the number of nodes and hidden layers in the system. Recently, deep learning exhibits suitable solutions in many flourishing fields based on different series of artificial neural networks for mapping nonlinear functions. To address the obstacles, this paper proposes an imaging method using an optimizer reconstruction model. An optimization model for imaging is generated as a powerful optimizer for building a computational model to ameliorate the reconstruction accuracy. Based on the deep learning methodology, the previous images reconstructed by using one of the imaging techniques to the required images are abstracted and stored in the deep learning machine, resulting in an error rate of $8.9 \%$, and this is considered good on ECT. Therefore, an artificial neural network of the capacitance (ANNoC) system is introduced to estimate capacitance measurements.
\end{abstract}

\section{Introduction}

One of the modern technologies that do not require surgical intervention is the electronic capacitance tomography technique that relies on the application of electric voltage only [1]. Its work is based on the principle of permitting permeability in different organisms differing in terms of permittivity. The component phase composition may vary in permittivity depending on the applied voltage [2]. A change in permittivity leads to a change in the measurement of capacitance which will reflect the distribution of the phase mixture contrition [3]. For this reason, we are using the algorithm of image reconstruction to build the visual distribution area inside the device (pipeline). Because the number of measured capacitances is distributed in a nonlinear fashion and is less than the number of pixels that make up the recovered image, the recovered image is therefore not smooth and needs improvement [4]. Any results from ECT are featured in sensitivity to their distribution. The image reconstruction algorithm is the key for opening the horizon in the field of ECT, so more image reconstruction process algorithms are needed. There are two types of algorithms for image reconstruction of ECT [5]: noniterative and iterative algorithms. Noniterative algorithms such as linear backprojection are quick and simple, but their precision is actually not satisfying. So this type of algorithm is used for qualitative purposes. Iterative algorithms such as Landweber, Tikhonov, and Newton-Raphson algorithms are widely used because of their smoothness and give satisfactory results.

ECT is a mixture of hardware and software systems and has proven its usefulness for that a greater number of studies investigated in this regard, and also there are a lot of opportunities to develop and innovate in this field [6]. 
Designing will be worthy in both hardware as a device and software algorithm of the image reconstruction method. The connection between hardware and software makes the ECT system as shown in Figure 1.

Images taken from ECT reflect the density and the system will respond according to the information collected by the sensors.

The term tomography represents the process of getting $2 \mathrm{D}$ sections crossed which are called tomograms. Existing studies take effect more than $2 \mathrm{D}$ with recent development. Some researchers improve the $3 \mathrm{D}$ structure and obtain image reconstruction more accurately while other researchers use other dimensions or domains (time domain) to become 4D [7].

Tomography is classified into two types, namely, indirect and direct, was according to human eyes not even like X-ray. But later measurement of the boundary of the object is included and used in image reconstruction [8]. For the indirect type of tomography, many physical quantities are implemented that use boundary measurement by using different tomography systems.

There are two criteria for an acceptable tomography algorithm from an engineering point of view [9]:

(i) Noninvasive: not requiring direct contact between the object or domain and sensors

(ii) Nonintrusive: keeping the object that explored not changing even not distributed in nature

More useful devices use the principles of ECT especially in medical and health fields, which used ECT perfectly and produced reconstructed images [10], as shown in Figure 2.

Three categories can be listed in the tomography system: (1) sensors, (2) data acquisition, and (3) reconstruction technique. The modality of tomography used can determine performance, sensors, and associated issues. In sensors classification, soft field with pose response determines the location of the imaging parts [11]. However, the problem in the data acquisition tomography is the power. If the number of sensors is low, then the signal-to-noise ratio (SNR) is low. To avoid this issue, the number of sensors is increased to cover more areas to get higher SNR.

In recent years, ECT has proved that it is a perfect method in industrial and medical fields due to many reasons such as nonradiation, high speed, and ability of nonintrusiveness [10]. It has been found that ECT has high temporal resolution when compared with other methods. Many of the electrophoresis engineering applications based on ECT suffer from image reconstruction, which has long been considered a challenge. A lot of studies consider this issue, and great results have been achieved in this regard by using the methods such as linear back-projection method [12], Tikhonov regulation technique [13], OIOR algorithm [11], Landweber algorithm [14], total variation method [15], and level set algorithm [16].

The manuscript is structured as follows. The methods and definitions are introduced in Section 1. Section 2 provides the literature review and explores the existing techniques used and the comprehensive structure of ECT to

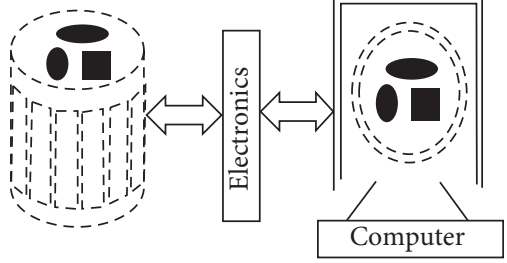

Figure 1: ECT system including H/W and S/W.

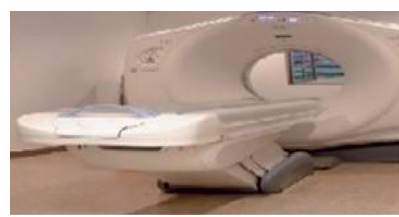

CT scan

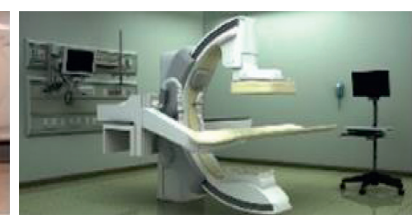

X-ray

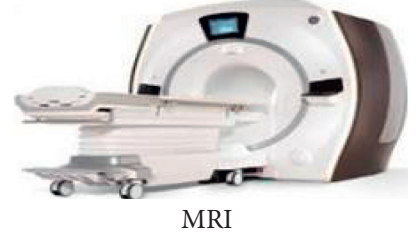

Figure 2: Devices that use ECT.

get better knowledge. It provides a better understanding of what deep learning is and describes the proposed method in detail. Section 3 discusses the results and findings, and finally, Section 4 provides the conclusion.

\section{Literature Review}

Image reconstruction in the ECT system has been developed using a deep learning algorithm with a big dataset based on moveable sensors [17]. Capacitance value is controlled under image reconstruction to measure the distribution of permittivity in ECT based on autoencoder [18]. Multilevel reconstruction is used in ECT to enable images suggested in [19] based on a deep neural network and images obtained via two pairs of electrodes for industrial issues. A data-driven plug-and-play prior abstracted (as presented in [20]) using deep convolutional neural is used to recover the image and high-dimensional permittivity distribution from degraded capacitance measured in the ECT system to achieve high computational issues. The method developed by Rymarczyk [21] includes multisource data of multisensors to get good image quality for reconstruction, and this method achieved good results and accuracy when applying in big data but the disadvantage is low accuracy when running in small data like a medical model.

2.1. Structure of ECT. The major component of ECT is the sensors as hardware, which is composed of $\boldsymbol{n}$ electrodes located around over the vessel (surrounding manner on the wall) as shown in Figure 3. It is necessary to determine the number of independent capacitances configured as $\mathbf{n}(\mathbf{n}-$ 1)/2 for management availability. 
The Poisson equation responsible for finding the distribution of permittivity related to the measurement of capacitance is as follows:

$$
\nabla \cdot(\varepsilon(x \cdot y) \nabla \varnothing(x \cdot y))=-p(x \cdot y),
$$

where $\varepsilon(x . y)$ is the permittivity distribution, $\varnothing(x . y)$ denotes the distribution of electrical potential, and $p(x . y)$ denotes the charge distribution. The Poisson equation is considered as a linear differential equation in the scope of $\varnothing(x . y)$. However, the nonlinear ECT results achieved by field distribution dependence $\varnothing(x . y)$ differ from the unknown solution $\varepsilon(x . y)$.

The mutual capacitance between the two opposite pairs of electrodes is defined as the ratio of stored charge [22] to the potential difference, which is given by

$$
C_{i j}=\frac{Q_{j}}{\Delta V_{i j}},
$$

where $C_{i j}$ is the mutual capacitance, $\Delta V_{i j}$ is the difference between $i$ electrode and $j$ electrode, $Q_{j}$ is the charge received by the electrode and can be found using Gauss law as follows:

$$
Q_{j}=\oint_{\Gamma_{i}} \varepsilon(x \cdot y) \nabla \varnothing(x \cdot y) \widehat{n} d l,
$$

where $\Gamma j$ is the closest enclosing path for detecting electrodes and $\hat{n}$ is the normal unit vector for $\Gamma j$. Combining equations (1) and (2) yields mutual capacitance as follows:

$$
C_{i j}=\frac{1}{\Delta V_{i j}} \oint_{\Gamma_{j}} \varepsilon(x \cdot y) \nabla \varnothing(x \cdot y) \cdot \hat{n} d l .
$$

This equation is considered as the general problem equation for ECT, where we can find capacitance for permittivity and boundary. Inversing this equation yields permittivity distribution.

2.2. Deep Learning. Current studies focus on automating industrial and medical devices. Making the machine touchable is not easy and requires powerful processors and high-performance computers. The development in data science and modern technology such as big data and highperformance computers provided an opportunity for machine learning to understand data and its behavior through complex systems. Machine learning gives the machine ability to learn different algorithms without strict orders from certain programs or limited instructions [23].

Deep learning can be defined as a technique of machine learning to learn useful features directly from given images, sound, and text. Many layers are exploited by deep learning for nonlinear data processing of unsupervised or supervised feature extraction for classification and pattern recognition [24]. Deep learning motivation is greatly reduced by artificial intelligence (AI) area, which simulates the ability of the human brain to analyze, make decisions, and learn. The deep learning goal is to emulate the approach of hierarchical

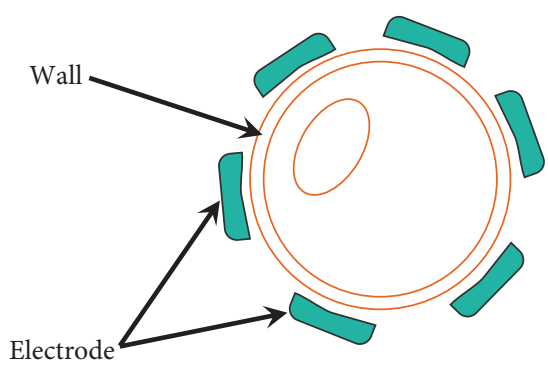

FIgURE 3: Structure of ECT that shows the electrodes.

learning of extracting features by the human brain directly from unsupervised data.

The core of deep learning is the hieratically computed features and representation of information, such as defining the features starting from low level to high level. With images, the standard techniques of machine learning are not working well when running directly due to the ignoring nature of image composition. In deep learning, features are extracted automatically from image reconstruction. The characteristics of this method of features are considered as one of the learning methodologies in the system [17].

Characterization of data acquisition when used as a feature is the key issue to the success of processing ECT. There is a limitation for extracted features in ECT such as power used within electrodes and the number of electrodes that organize the data [25]. For this reason, we can use deep learning by its feature extraction to solve limitations in ECT.

The main difference between machine learning and deep learning is the feature selection method, as shown in Figure 4.

Features in deep learning will be generated automatically to simulate the appropriate results [13]. Different hidden layers participated in decision-making by using the feedback from one next layer to the previous one or the resulting layer will have fed into the first layer [26]. DL enables computers to be able to perform complex calculations by relying on simpler calculations to optimize computer efficiency. It is difficult for a computer to understand complex data such as the number of electrodes with their directions or a series of data of a complex nature, so we use deep learning algorithms instead of usual learning methods [27].

First, deep learning considers the nonlinear relationship between permittivity and capacitance that determines the quality of image reconstructed depending on the linear model since the variation of permittivity is getting large. This actually due to the linear model is similar to the nonlinear relationship between the corresponding permittivity and data capacitance [13]. For this reason, DNN is used to solve the nonlinear model for improving the quality of image reconstruction. The second factor that deep learning took into consideration is speed. Real time is necessary for online imaging during data acquisition and image reconstruction.

The ECT process includes two main parts which are taken into consideration in the proposed method. It is one completely opposite to the other, while the process of retrieving and transferring data to the system is considered as a 


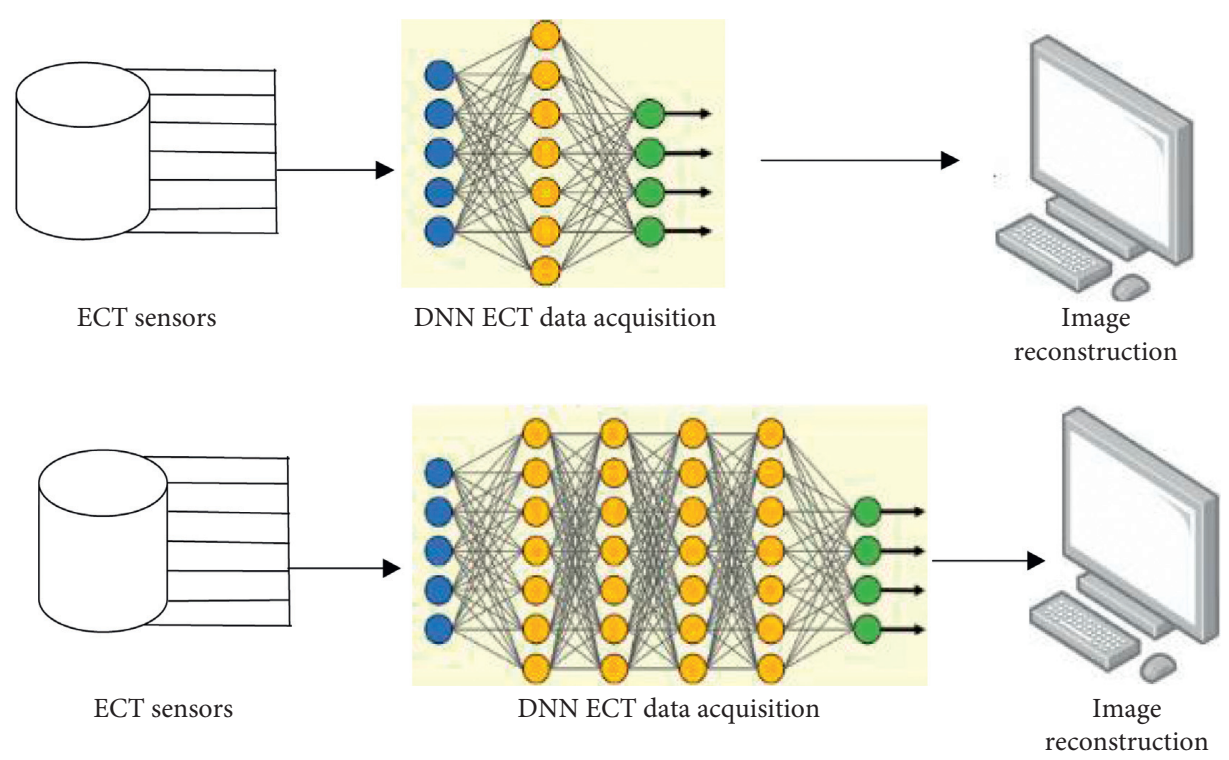

FIGURE 4: Difference between machine learning and deep learning in feature combination techniques.

coding process, and in return, the data are used to reconstruct an image of the form from which the data were extracted called decoding.

Suppose $x$ is the vector of permittivity distribution, $y$ is the vector of capacitance data, $\hat{x}$ is the distribution of reconstructed permittivity, and $\hat{y}$ is the estimated data capacitance which is calculated by the distribution of permittivity [28]. Then, the autoencoder (Figure 5) (code and decode) behave like

$$
\left\{\begin{array}{l}
\hat{y}=F(x) \\
\hat{x}=G(y)
\end{array} .\right.
$$

As shown in Figure 5, there are two contrary problems: encode called ECT forward problem and decode called inverse ECT problem; these are under the autoencode framework. There are two main terms in deep neural network especially when related to a subject like ECT: encoding which gathers the informative data and converts to input data and then after processing data will be converted to structured data as decoding issue. There are two vectors derived from autoencode framework $\vec{x}$ and $\vec{y}$ defined as follows:

$$
\left\{\begin{array}{l}
\vec{y}=F(\widehat{x})=F(G(y)) \\
\vec{x}=G(\widehat{y})=G(F(x))
\end{array}\right.
$$

2.3. Proposed Method. Image reconstruction is considered the main issue in ECT; method that improves the technique will reflect directly to the resulting image reconstruction. These reconstructions are based on the signals that come from the electrodes distributed over the pipelining. Increasing the number of electrodes gives high resolution to the image which gets better detection and recognition. When changing the structure of electrodes, better measurement can be achieved according to the voltage and I signal.

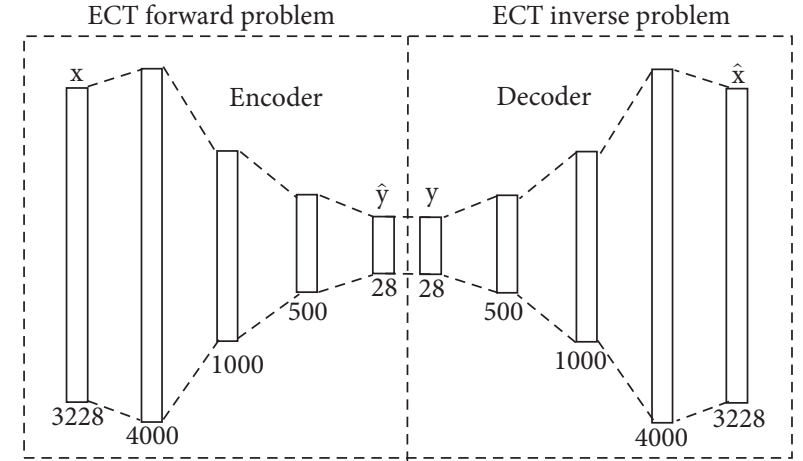

Figure 5: Structure of ECT with deep encoder and decoder.

The issue here is to measure the parameters so that machine can learn to conclude the way of solving problems with best result in term of subject. ECT consists of three units as illustrated in Figure 6.

In order to compare in measurement section, the hardware of the acquisition data device has to be fixed. All the measurements are based on this type and according to architecture design. The following equation illustrates the parameters in detail which reflects the measurement value:

$$
C=\frac{Q}{\Delta V}, C=\frac{\varepsilon_{0} \varepsilon_{r} A}{d},
$$

where $C$ is the capacitance required, $Q$ is the amount of charge, $\varepsilon_{0}$ is the vacuum permittivity, $\varepsilon_{r}$ is the material permittivity, and $A$ is the geometry of the area. $\Delta V$ is the potential difference between voltage and distance $d$ between the two electrodes.

These parameters must be considered during image reconstruction where the system takes the effect of the reverse process in order to get retrieval data as following equations: 


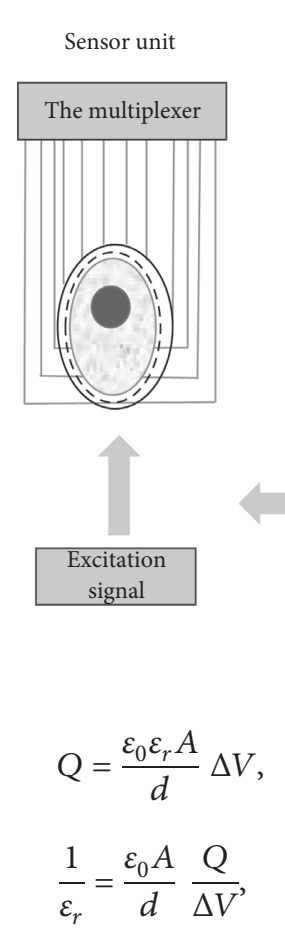

If we know $\Delta V$, we can easily estimate the permittivity, or in contrast, when we know the amount of charge $Q$, we can find variance voltage applied inside the pipe. The structure of the sensors or acquisition sensor is explained in Figure 7.

Increasing the number of electrodes allows reconstructing in high resolution. The voltage that passes through the electrodes makes magnetic flux penetrate the objects and by observing the remaining gives the appropriate image reconstructed to illustrate the object from outside. Table 1 shows the number of electrodes with corresponding measurements.

Signal when delivered to ECT started to manipulate within the neural network process and was stored in the preprocessing stage. Reconstructed image within noncircular electrodes in the noncircular region must be ignored, because of the mismatch of the pixels in the reconstructed image with the signal coming from the sensor and it must be processed for normalization. There are two steps to calculate the number of pixels in the image: first, signals with fixed features such as the number of path measurements are classified into one hidden layer, and second, data that occur outside the noncircular region will be in another hidden layer, so for the next iteration, the system can conclude the necessary hidden layer that will add.

For given pixels of image $Q(x, y)$ to be used with wavelet $g(x, y)$,:

$$
R_{m n}(x \cdot y)=\int Q(x \cdot y) g_{m n}^{*}\left(x-x_{1} \cdot y-y_{1}\right) d x_{1} d y_{1} .
$$

For example, $R_{m n}(x . y)$ is the response filter with coordinate $(x, y)$ and $m$ and $n$ that scale from 1 to $\mathrm{M}$ and $\mathrm{N}$ sequentially representing the number of scales and orientations, respectively. Standard deviation and response filter

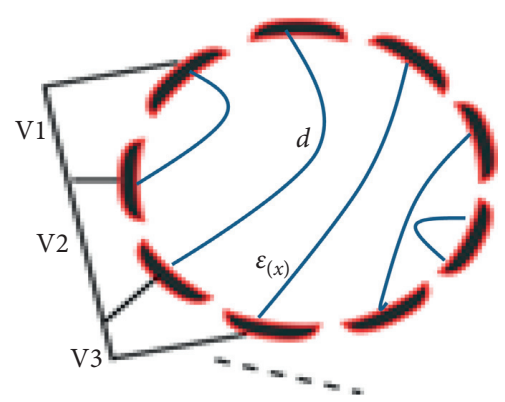

FIgURE 7: Structure of sensors with their parameters.

TABLE 1: Electrodes of the sensor with corresponding paths and frame rates.

\begin{tabular}{lcc}
\hline Electrodes & Measurements (paths) & Frame rate $(\mathrm{Hz})$ \\
\hline 2 & 82 & 10 \\
16 & 120 & 2.3 \\
32 & 469 & 0.6 \\
\hline
\end{tabular}

can be used to represent the region derived from clustering of region pixels.

$$
\begin{aligned}
\mu_{m n} & =\iint\left|R_{m n}(x \cdot y)\right| d x d y, \\
\sigma_{m n} & =\sqrt{\iint\left(\left|R_{m n}(x \cdot y)\right|-\mu_{m n}\right)^{2} d x d y .}
\end{aligned}
$$

Features collected in the vector are called feature vector $\overrightarrow{V_{f}}$

that was constructed using HT descriptor as follows:

$$
H T=\left[\mu_{11} \sigma_{11} \pi_{12} \sigma_{12}, \ldots, \mu_{M N} \sigma_{M N}\right] .
$$

Texture feature from regional image starts as filter of channels from given image then combined with other features.

The proposed algorithm working with adaptive features and variable parameters to achieve training and testing modes is illustrated in Figure 8.

Training mode starts with knowing results in advance to adjust parameters that control the hidden layers. In Figure 8, 


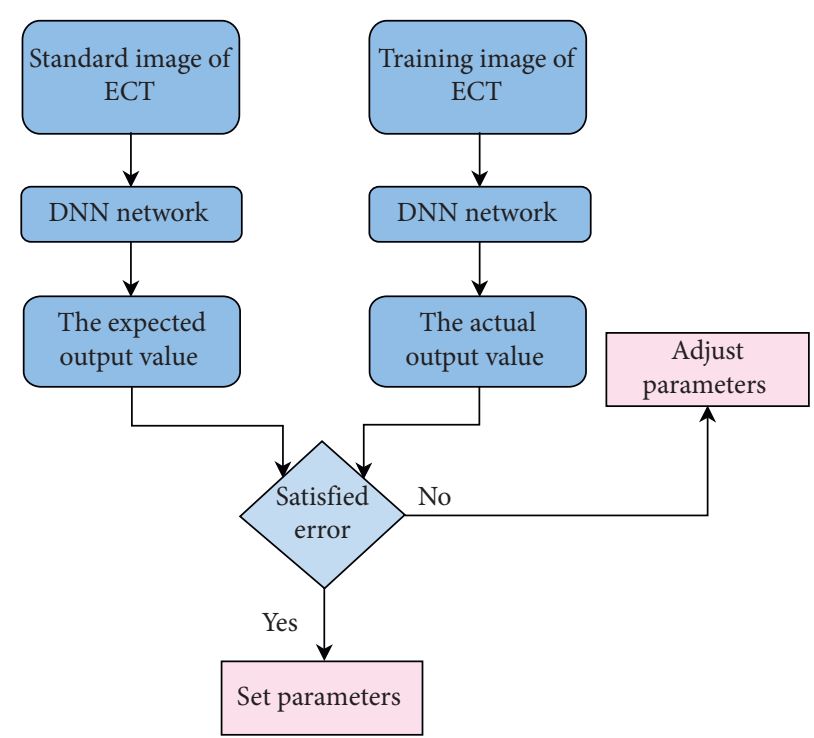

FIGURE 8: Standard proposed flowchart for the system.

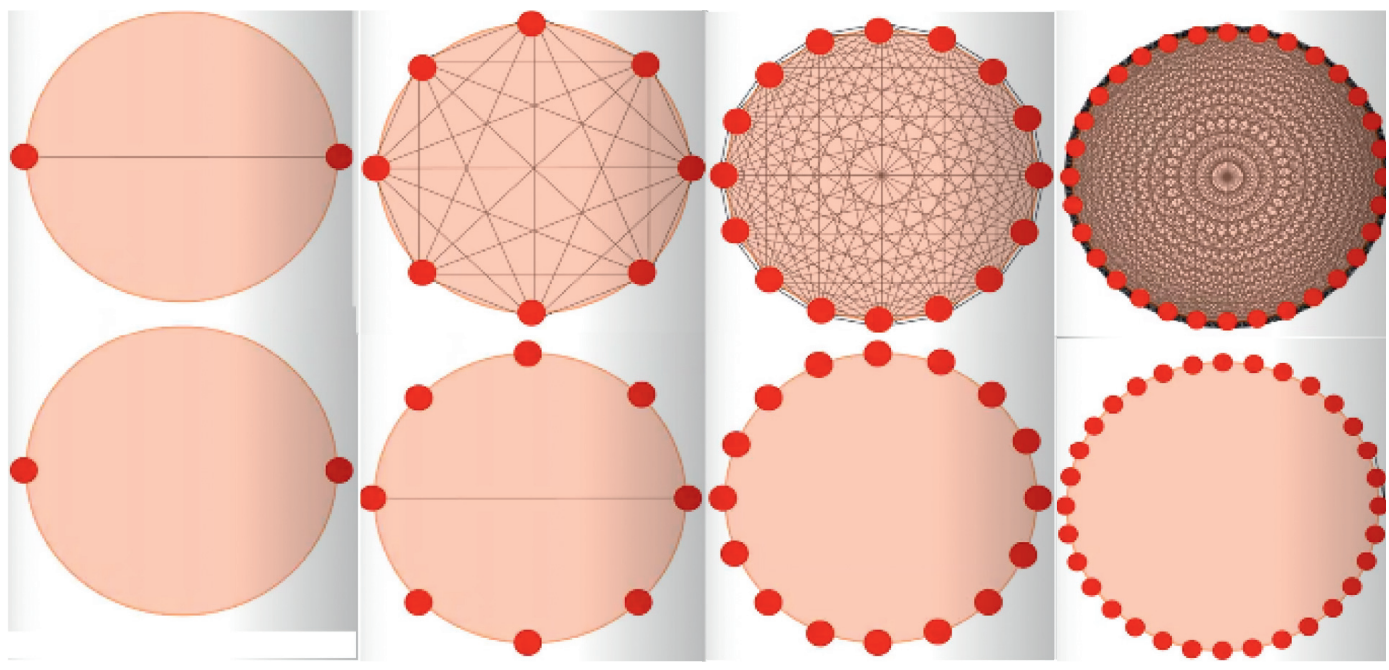

FIgURe 9: Distribution of the located electrodes over the pipe.

stages in the right represent training with labeled data while stages in the left represent testing with known labeled data (expected) and this is the purpose of the system. The number of hidden layers increases or decreases accordingly with results during the training mode. The actual data come from testing through DNN to expect the results and process running many times as iterations till satisfactory results are obtained, which will be discussed in the next section.

\section{Experimental Results}

Many applications used the term ECT, and most of these applications required accurate results especially in industry and medical fields. Evaluation of such system is important to reduce the mean square error (MSE), and the evaluation of the proposed method evaluation needs more accuracy because of having two strategies: testing and training modes. Training mode starts with known results given from the standard dataset, while actual running goes through the real data in different fields. Start with sensors that consider data acquisition represented by the pipelining supported with a certain number of electrodes. Figure 9 shows the distribution of the electrodes with corresponding paths to create intersection fields.

The bottom line represents the located electrodes $2,8,16,32$ with corresponding associated paths 4,82,120, 469, respectively, as shown in Table 2 of resulting parameters.

Another test mode for image reconstruction is the true distribution in experimental data since the capacitance error was evaluated above. The reconstructed image appears to have a improved resolution in the central part. Results show that image within deep learning is strongly parameter-dependent as shown in Figure 10.

Within the true distribution of the testing system, Figure 11 shows that in the first row of the image, 1 rod is located at the center of the first square on the left, then the 
TABLE 2: Results of training mode with 32 electrodes for the ECTY system with 65 iterations with image error.

\begin{tabular}{lcccc}
\hline Pattern & Relative image error (\%) & Correlation coefficient & Estimate phase ratio (\%) & Phase ratio error (\%) \\
\hline $50 \%$ annular & 37.30 & 0.95 & 47.5 & -2.75 \\
$19.58 \%$ stratified & 40.19 & 0.90 & 17.67 & -1.91 \\
$13.88 \%$ single bar & 84.5 & 0.65 & 7.30 & -6.59 \\
$27.76 \%$ two bars & 72.04 & 0.71 & 17.2 & 10.24 \\
\hline
\end{tabular}

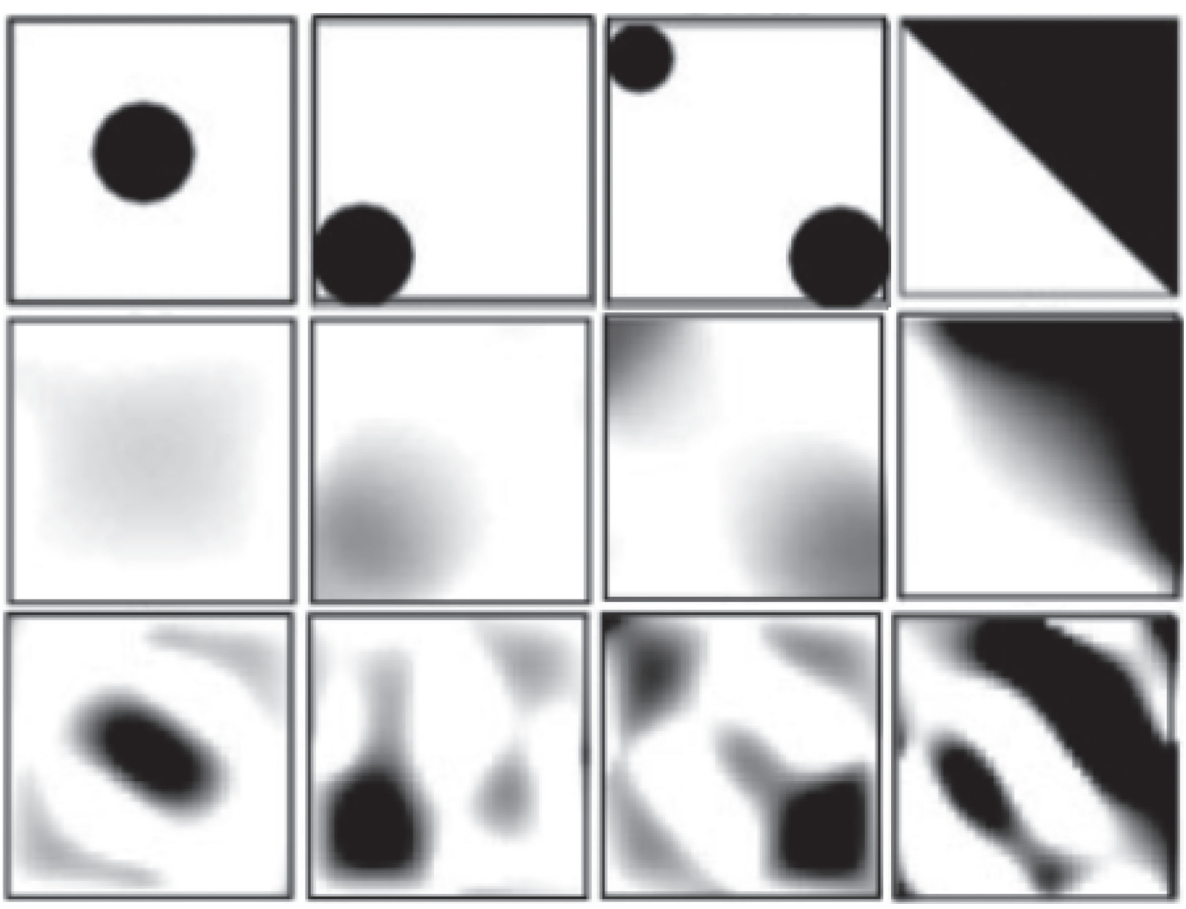

FIgURE 10: Image reconstruction with four cases.

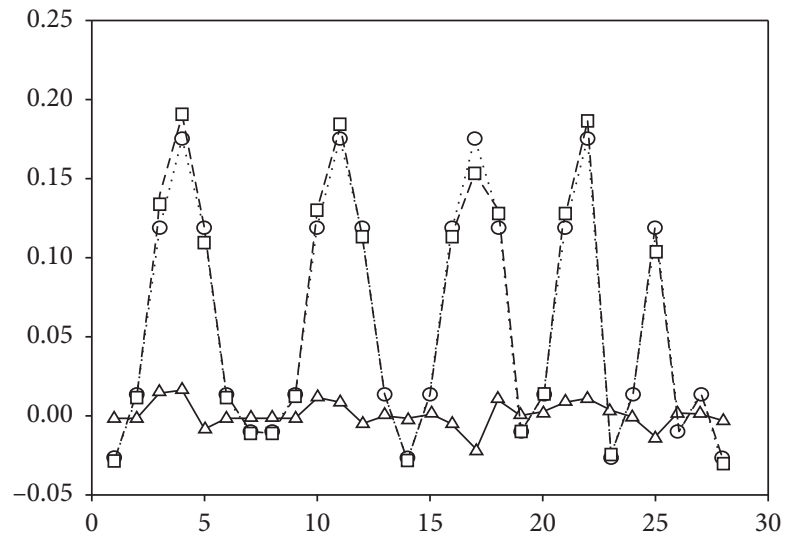

. O. True capacitance vector corresponding to test phantom

- $\mathrm{-}$ - Capacitance vector contaminated by noise

$\checkmark$ Noise vector

FIGURE 11: Capacitance vector with noise.

rod is moved near the wall of the bottom left, and after that, two rods are distributed on the corner and then stratified. The next two rows represent the corresponding image reconstructed by ECT.

To investigate the influence of error on the system of image reconstruction, a random error was added to the vector of capacitance. So noise $\lambda$ was added to the reconstruction vector to test the sensitivity of the system and then calculated using permittivity distribution. The capacitance vector that was contaminated with noise $\lambda$ was added randomly to get distributed noise $\bar{\lambda}$, then the error between them is defined as $\lambda-\bar{\lambda} / \bar{\lambda}$, resulting in $8.9 \%$, as shown in Figure 10.

The worthiness of the system comes from modifying parameters within new suggested parameters by hidden layers to increase both accuracy and image reconstructed resolution.

\section{Conclusion, Limitations, and Future Work}

Many applications are based on electronic capacitance tomography (ECT). It is important for the investigation and development of such systems to take place. The ECT system relies on the ability to reconstruct an image from the signals obtained via the data acquisition device called sensors. The system consists of three main parts: sensors, measuring signals, and image reconstruction. In this study, we considered collecting incoming signals from data acquisition device (sensors) to manipulate and build a deep neural network for learning ECT. Increasing the resolution of the reconstructed image is the main purpose of this study, and thus, DNN tried to process the parameters and train the 
system for that. Achieved results prove the worthiness of the proposed method. One of the purposes of such method is to open the horizon for more investigation in terms of machine learning. For future work, we recommend extracting more informative data and train the system with a large dataset to learn the system in addition to increase the number of sensors to allow the system to gain high resolution.

\section{Data Availability}

All data used to support the findings of this study are included within the article.

\section{Conflicts of Interest}

The authors confirm that there are no conflicts of interest regarding this study.

\section{Acknowledgments}

The authors extend their appreciation to National Natural Science Foundation of China (grant numbers 60572153 and 60972127), Special Research Fund for Doctoral Program of Higher Education (grant number 200802140001), Natural Science Foundation of Heilongjiang Province (grant number QC2012C059), Harbin Science and Technology Innovation Talents Research Fund (grant number 2014RFXXJ022), and Science and Technology Research Project of Education Department of Heilongjiang Province (grant numbers 11541040 and 12531094) for funding and supporting this work.

\section{References}

[1] S. M. Chowdhury, Q. M. Marashdeh, and F. L. Teixeira, "Electronic scanning strategies in adaptive electrical capacitance volume tomography: tradeoffs and prospects," IEEE Sensors Journal, vol. 20, no. 16, pp. 9253-9264, 2020.

[2] H. Wang and W. Yang, "Application of electrical capacitance tomography in circulating fluidised beds - a review," Applied Thermal Engineering, vol. 176, p. 115311, 2020.

[3] Z. Cui, Y. Chen, and H. Wang, "A dual-modality integrated sensor for electrical capacitance tomography and electromagnetic tomography," IEEE Sensors Journal, vol. 19, no. 21, pp. 10016-10026, 2019.

[4] M. Majchrowicz, P. Kapusta, L. Jackowska-Strumiłło, R. Banasiak, and D. Sankowski, "Multi-GPU, multi-node algorithms for acceleration of image reconstruction in $3 \mathrm{D}$ Electrical Capacitance Tomography in heterogeneous distributed system," Sensors, vol. 20, no. 2, p. 391, 2020.

[5] S. Wang, X. Sun, C. Xu, J. Bao, C. Peng, and Z. Tang, "Investigation of a circulating turbulent fluidized bed with a fractal gas distributor by electrostatic-immune electrical capacitance tomography," Powder Technology, vol. 361, pp. 562-570, 2020.

[6] Q. Guo, M. Ye, W. Yang, and Z. Liu, "A machine learning approach for electrical capacitance tomography measurement of gas-solid fluidized beds," AIChE Journal, vol. 65, no. 6, p. e16583, 2019.

[7] A. Voss, P. Hosseini, M. Pour-Ghaz, M. Vauhkonen, and A. Seppänen, "Three-dimensional electrical capacitance tomography - a tool for characterizing moisture transport properties of cement-based materials," Materials \& Design, vol. 181, p. 107967, 2019.

[8] T. Xia, H. Xie, A. Wei, R. Zhou, L. Qiu, and X. Zhang, "Preliminary study on three-dimensional imaging of cryogenic two-phase flow based on electrical capacitance volume tomography," Cryogenics, vol. 110, p. 103127, 2020.

[9] J. Lei, Q. B. Liu, X. Y. Wang, and R. S. Yan, "Distributed electrical capacitance tomography reconstruction with data and sparsity priors," Signal Processing, vol. 181, p. 107922, 2021.

[10] W. Yang, Z. Ren, M. Takei, and J. Yao, "Medical applications of electrical tomography," in Proceedings of the 2018 IEEE International Conference on Imaging Systems and Techniques (IST), Krakow, Poland, October 2018.

[11] J. Lei, H. P. Mu, Q. B. Liu, X. Y. Wang, and S. Liu, "Datadriven reconstruction method for electrical capacitance tomography," Neurocomputing, vol. 273, pp. 333-345, 2018.

[12] J. Zheng and L. Peng, "A deep learning compensated back projection for image reconstruction of electrical capacitance tomography," IEEE Sensors Journal, vol. 20, no. 9, pp. 4879-4890, 2020.

[13] H. Zhu, J. Sun, L. Xu, W. Tian, and S. Sun, "Permittivity reconstruction in electrical capacitance tomography based on visual representation of deep neural network," IEEE Sensors Journal, vol. 20, no. 9, pp. 4803-4815, 2020.

[14] M. Xie, H. Yang, K. Zhao et al., "Regularization parameter optimization based on the constraint of Landweber algorithm for electrical capacitance tomography," Flow Measurement and Instrumentation, vol. 69, no. 4, p. 101620, 2019.

[15] K. Li, T. C. Chandrasekera, Y. Li, and D. J. Holland, "A nonlinear reweighted total variation image reconstruction algorithm for electrical capacitance tomography," IEEE Sensors Journal, vol. 18, no. 12, pp. 5049-5057, 2018.

[16] J. Lei, Q. B. Liu, X. Y. Wang, and S. Liu, "Combination regularization reconstruction method for electrical capacitance tomography," Flow Measurement and Instrumentation, vol. 59, pp. 135-146, 2018.

[17] J. Zheng, J. Li, Y. Li, and L. Peng, "A benchmark dataset and deep learning-based image reconstruction for electrical capacitance tomography," Sensors, vol. 18, no. 11, p. 3701, 2018.

[18] J. Zheng and L. Peng, "An autoencoder-based image reconstruction for electrical capacitance tomography," IEEE Sensors Journal, vol. 18, no. 13, pp. 5464-5474, 2018.

[19] E. Chen and C. D. Sarris, "A multi-level reconstruction algorithm for electrical capacitance tomography based on modular deep neural networks," in Proceedings of the 2019 IEEE International Symposium on Antennas and Propagation and USNC-URSI Radio Science Meeting, Atlanta, GA, USA, July 2019.

[20] J. Lei, Q. B. Liu, and X. Y. Wang, "Computational imaging method with a learned plug-and-play prior for electrical capacitance tomography," Cognitive Computation, vol. 12, no. 1, pp. 206-223, 2020.

[21] T. Rymarczyk, "Application of multi-source data for process analysis in electrical tomography," Przeglad Elektrotechniczny, vol. 1, no. 12, pp. 194-197, 2019.

[22] K. Huang, S. Meng, Q. Guo et al., "Effect of electrode length of an electrical capacitance tomography sensor on Gas-Solid fluidized bed measurements," Industrial \& Engineering Chemistry Research, vol. 58, no. 47, pp. 21827-21841, 2019.

[23] M. López de Prado, Advances in Financial Machine Learning, John Wiley \& Sons, Hoboken, NJ, USA, 2018.

[24] G. Sulong and A. Mohammedali, "Human activities recognition via features extraction from skeleton," Journal of 
Theoretical \& Applied Information Technology, vol. 68, p. 3, 2014.

[25] G. Sulong and A. Mohammedali, "Recognition of human activities from still image using novel classifier," Journal of Theoretical \& Applied Information Technology, vol. 71, p. 1, 2015.

[26] B. T. Atiyha, S. Aljabbar, A. Ali, and A. Jaber, "An improved cost estimation for unit commitment using back propagation algorithm," Malaysian Journal of Fundamental and Applied Sciences, vol. 15, no. 2, pp. 243-248, 2019.

[27] D. Xie, L. Zhang, and L. Bai, "Deep learning in visual computing and signal processing," Applied Computational Intelligence and Soft Computing, vol. 2017, Article ID 1320780, 2017.

[28] G. Guo, G. Tong, L. Lu, and S. Liu, "Iterative reconstruction algorithm for the inverse problems in electrical capacitance tomography," Flow Measurement and Instrumentation, vol. 64, pp. 204-212, 2018. 